\title{
SINGULAR POINT PROBLEMS IN THE THEORY OF LINEAR DIFFERENTIAL EQUATIONS $†$
}

\author{
W. J. TRJITZINSKY
}

1. Introduction. The discussion of the subject indicated in the title is not intended in any way to be encyclopaedic. The object of this address relates to the general problems of determining the character of solutions of equations

(A) $L_{n}(y) \equiv a_{0}(x) y^{(n)}+a_{1}(x) y^{(n-1)}+\cdots+a_{n-1}(x) y^{(1)}+a_{n}(x) y=0$,

(B) $L_{n}(x, \lambda ; y) \equiv a_{0}(x, \lambda) y^{(n)}+a_{1}(x, \lambda) y^{(n-1)}+\cdots+a_{n}(x, \lambda) y=0$.

The $a_{i}(x)$ in (A) are assumed to be analytic for $|x| \geqq \rho,(x \neq \infty)$, being representable by convergent series of the form

$$
\begin{aligned}
& \alpha_{i}(x)=x^{n_{i} / p} {\left[a_{i, 0}+a_{i, 1} x^{-1 / p}+a_{i, 2} x^{-2 / p}+\cdots\right], } \\
& n_{i}, p \text { integers } ; p>0 ; i=0, \cdots, n ;|x| \geqq \rho,
\end{aligned}
$$

or they are supposed to be merely asymptotic to such possibly divergent series, for $x$ in a suitable region extending to infinity. The $a_{i}(x, \lambda)$ in (B) are assumed to be indefinitely differentiable in $x$, for $x$ on a closed real interval $(a, b)$, and analytic in $\lambda$ for $|\lambda| \geqq \rho>0$, $(\lambda \neq \infty ; \lambda$ is a parameter), being representable by convergent series of the form

$$
\begin{gathered}
\alpha_{i}(x, \lambda)=\lambda^{n_{i}} \sum_{\nu=0}^{\infty} \alpha_{i, \nu}(x) \lambda^{-\nu}, \quad n_{i} \text { integers; } i=0, \cdots, n ; \\
\alpha_{i, \nu}(x) \text { indefinitely differentiable on }(a, b) ; a \leqq x \leqq b ;|\lambda| \geqq \rho,
\end{gathered}
$$

or they are supposed to be asymptotic to a finite number of terms, or to infinitely many terms, to such possibly divergent series, when $x$ is on $(a, b)$ and the parameter $\lambda$ is in a suitable region $R$ extending to infinity.

The investigation for the problems (A) and (B) (relating to equations (A) and (B), respectively) has the purpose of establishing the character of solutions in the complex neighborhood of the singular point of the equation under consideration. In problem (A) the singular point is at $x=\infty$. In problem (B) the singular point considered is at $\lambda=\infty,(x$ in $(a, b))$.

$\dagger$ An address delivered by invitation of the Program Committee at the Iowa City Meeting of the Society, November 26, 1937. 
These problems are important, in part, because many significant classical instances of differential equations occur as particular cases of (A) and (B); on the other hand, a satisfactory theory of problems (A) and (B) is a very desirable prerequisite for the analytic theories of certain highly important non-linear differential systems.

We use the term asymptotic relation in the following sense. If $R$ is a region extending to infinity in the complex $x$ plane it will be said that a function $a(x)$, defined in $R$, is asymptotic to $\alpha(x)=a_{0}+a_{1} x^{-1 / p}+\cdots$ for $x$ in $R$ (that is, $a(x) \sim \alpha(x)$ in $R$ ) if

$$
\begin{aligned}
& a(x)=a_{0}+\cdots+a_{m-1} x^{-(m-1) / p}+\beta_{m}(x) x^{-m / p}, \\
& \qquad\left|\beta_{m}(x)\right| \leqq \beta_{m} \text { in } R ; m=1,2, \cdots .
\end{aligned}
$$

If $R$ is a region extending to infinity in the complex $\lambda$ plane, we shall say that a function $a(x, \lambda)$, defined for $x$ in $(a, b)$ and $\lambda$ in $R$, is asymptotic to $\alpha(x, \lambda)=\alpha_{0}(x)+\alpha_{1}(x) \lambda^{-1 / p}+\cdots$, (integer $p>0$ ), in $R$ (with $x$ on $(a, b)$ ) if the $\alpha_{\nu}(x)$ are continuous on $(a, b)$ (which interval is taken closed) and

$$
a(x, \lambda)=\alpha_{0}(x)+\cdots+\alpha_{m-1}(x) \lambda^{-(m-1) / p}+\beta_{m}(x, \lambda) \lambda^{-m / p},
$$

$\left|\beta_{m}(x, \lambda)\right| \leqq \beta_{m} ; \beta_{m}$ independent of $x, \lambda ; m=1,2, \cdots ; x$ in $(a, b) ; \lambda$ in $R$.

Unless the contrary is stated the designation

$$
a(x) \sim \alpha(x) \text { in } R, \quad a(x, \lambda) \sim \alpha(x, \lambda), \quad x \text { in }(a, b) ; \lambda \text { in } R,
$$

will indicate asymptotic relations in the sense that (1.3), (1.4) hold in the respective cases. Such asymptotic relations will be termed ordinary or to infinitely many terms. When (1.3) (or (1.4)) is asserted only for $m=1,2, \cdots, m_{0}$, then we shall write

$$
\text { (1.5a) } \quad a(x){\widetilde{m_{0}}}_{\alpha} \alpha(x) \text { in } R \quad \text { (or } a(x, \lambda){\widetilde{m_{0}}}_{\alpha} \alpha(x, \lambda), x \text { in }(a, b) ; \lambda \text { in } R \text { ), }
$$

the symbol $\widetilde{m}_{0}$ denoting an asymptotic relation "to $m_{0}$ terms."

In consequence of a paper by E. Fabryt it can be asserted that the formal equation ( $\mathrm{A}^{*}$ ) obtained from (A) by replacing the $a_{i}(x)$ by the corresponding series $\alpha_{i}(x)$ (of (1.1)) $\ddagger$ has $n$ formally linearly independent solutions

$$
s_{i}(x)=e^{Q i(x)} x^{r i} \sigma_{i}(x), \quad i=1, \cdots, n,
$$

where the $Q_{i}(x)$ are polynomials in $x^{1 / k_{i}}$ and

$\dagger \mathrm{E}$. Fabry, Sur les intégrales des équations différentielles linéaires à coefficients rationnels, Thèse, 1885, Paris.

$\ddagger$ It is assumed that in $\alpha_{0}(x)$ not all the coefficients are zero. This hypothesis is made for convenience and has no essential significance. 


$$
\begin{aligned}
& \sigma_{i}(x)=\sigma_{i}{ }^{0}(x)+\sigma_{i}{ }^{1}(x) \log x+\cdots+\sigma_{i}{ }^{m}(x) \log ^{m_{i}} x, \\
& \sigma_{i}^{\gamma}(x)=\sigma_{i}^{\gamma, 0}+\sigma_{i}^{\gamma, 1} x^{-1 / k_{i}}+\cdots, \\
& \gamma=0, \cdots, m_{i} ; i=1, \cdots, n ;
\end{aligned}
$$

here $m_{i}, k_{i}$ are integers $\left(m_{i} \geqq 0 ; k_{i}(>0)\right.$ is an integral multiple of $\left.p\right)$, and the series (1.6b) may diverge (for all $x$ ).

In consequence of the work of $\mathrm{P}$. Noaillon the formal equation $\left(\mathrm{B}^{*}\right)$, obtained from (B) by replacing the $a_{i}(x, \lambda)$ by the corresponding series $\alpha_{i}(x, \lambda)$ (of (1.2)), possesses a full set of $n$ formal (in general divergent) series solutions

$$
s_{i}(x, \lambda)=e^{Q_{i}(x, \lambda)} \sigma_{i}(x, \lambda), \quad i=1, \cdots, n,
$$

where the $Q_{i}(x, \lambda)$ are polynomials in $\lambda^{1 / k_{i}}$ (integer $k_{i}>0$ ) and

$$
\sigma_{i}(x, \lambda)=\sum_{\nu=0}^{\infty} \sigma_{i, \nu}(x) \lambda^{-\nu / k_{i}}, \quad i=1, \cdots, n,
$$

the coefficients in $Q_{i}(x, \lambda)$ and in the series $\sigma_{i}(x, \lambda)$ being functions indefinitely differentiable with respect to $x$ for $x$ in a suitable interval $(a, b)$.

The essential features of an analytic theory of equations (A) and (B) relate to the following questions.

I. If the $a_{i}(x)$ (or $a_{i}(x, \lambda)$ ) are representable by convergent series $\alpha_{i}(x)$ of (1.1) (or series $\alpha_{i}(x, \lambda)$ of (1.2)), under what conditions do the formal series solutions (all or any) converge, thus representing "actual" solutions of (A) (or of (B))?

II. If the $a_{i}(x)$ (or $a_{i}(x, \lambda)$ ) are representable by convergent series of the type stated above, under what conditions are the formal solutions, all or any, (1.6) (or (1.7)), though possibly divergent, summable to "actual" solutions?

With respect to II a method which is particularly potent is that involving what essentially amounts to exponential summabilityto be precise, Laplace integrals leading to expressions containing convergent factorial series. $\$$

$\dagger$ P. Noaillon, Développements asymptotiques dans les équations différentielles linéaires a paramètre variable, Mémoires de la Société des Sciences de Liège, (3), vol. 9 (1912), 197 pages.

$\ddagger$ Possibly merely a sub-interval of the interval originally so denoted.

$\S$ Developments of this type rest on important results obtained by N. E. Nörlund in the theory of factorial series (Nörlund applies these methods to difference equations); cf. N. E. Nörlund, Acta Mathematica, vol. 37 (1914), pp. 327-387; Leçons sur les Équations Linéaires aux Différences Finies, Paris, 1929. References to J. Horn's applications of methods mentioned in II to differential equations will be given later. 
III. If the $a_{i}(x)$ (or $a_{i}(x, \lambda)$ ) are representable as specified in I and II, but the methods suggested therein (that is, convergence and summability) fail, what can be done, in so far as "actual" solutions are concerned, by asymptotic methods? When the $a_{i}(x)$ (or $a_{i}(x, \lambda)$ ) are merely asymptotic to series of the type specified above, questions I, II lose their significance and only question III remains.

This address relates mainly to my own work regarding questions II and III. $\dagger$ Of my three papers, just referred to, $\left(\mathrm{T}_{1}\right)$ and $\left(\mathrm{T}_{2}\right)$ relate to question III for the problems (A) and (B), respectively. $\left(\mathrm{T}_{3}\right)$ relates to question II for the problem (A). $\ddagger$ From a certain point of view $\left(T_{1}\right),\left(T_{2}\right),\left(T_{3}\right)$ give a complete treatment of certain essential aspects of the theory of equations (A) and (B). The most relevant predecessors to these papers are certain contributions of a number of mathematicians. Of the predecessors to $\left(T_{1}\right)$ (relating to question III for the problem (A)), highly significant are papers of $\mathrm{H}$. Poincaré $\S$ and G. D. Birkhoff.|| With respect to $\left(\mathrm{T}_{2}\right)$ (relating to question III for the problem (B)) should be mentioned important developments due to G. D. Birkhoff, 9 as well as certain contributions due to Noaillon (already referred to) and to J. D. Tamarkin.** In connection with $\left(\mathrm{T}_{3}\right)$ (relating to question II for $(\mathrm{A})$ ) of the relevant predecessors we shall mention J. Horn. $\dagger \dagger$

\section{Problem (A) (asymptotic methods). H. Poincaré treats equation}

$\dagger$ W. J. Trjitzinsky, Analytic theory of linear differential equations, Acta Mathematica, vol. 62 (1934), pp. 167-226. Referred to as $\left(\mathrm{T}_{1}\right)$.

W. J. Trjitzinsky, Theory of linear differential equations containing a parameter, Acta Mathematica, vol. 67 (1936), pp. 1-50. Referred to as $\left(\mathrm{T}_{2}\right)$.

W. J. Trjitzinsky, Laplace integrals and factorial series in the theory of linear differential and linear difference equations, Transactions of this Society, vol. 37 (1935), pp. 80-146. Referred to as $\left(\mathrm{T}_{3}\right)$.

$\ddagger$ For the problem (B) summability methods, on the whole, appear to be rather ineffective.

$\S$ H. Poincaré, American Journal of Mathematics, vol. 7 (1885), pp. 203-258.

\| G. D. Birkhoff, Transactions of this Society, vol. 10 (1909), pp. 436-470.

9 G. D. Birkhoff, On the asymptotic character of solutions of certain linear differential equations, Transactions of this Society, vol. 9 (1908), pp. 219-231; also cf. ibid., vol. 9 (1908), pp. 373-395; also see G. D. Birkhoff and R. E. Langer, The boundary problems and developments ... , Proceedings of the American Academy of Arts and Sciences, vol. 58 (1923), pp. 51-128.

** J. D. Tamarkin, Some general problems of the theory of ordinary linear differential equations . . . Mathematische Zeitschrift, vol. 27 (1927), pp. 1-54.

$\dagger$ †ome of Horn's work is as follows: Integration linearer Differentialgleichungen ..., Jahresbericht der Deutschen Mathematiker-Vereinigung, vol. 24 (1915), pp. 309-329; Laplacesche Integrale . . , Mathematische Zeitschrift, vol. 21 (1924), pp. 85-95. 
(A), ( $\$ 1)$ of rank one, with the roots of the characteristic equation distinct. $\dagger$ G. D. Birkhoff's work relates to equation (A), (\$1) of any rank, the roots of the characteristic equation being distinct. $\ddagger$ The methods of these two writers are not readily extensible to the general problem (A).

In $\left(\mathrm{T}_{1}\right)$, with the aid of "iterations," factorizations, and appropriate integrations, is established a fundamental existence theorem for the general problem (A). To state this theorem conveniently a number of definitions will be introduced.

Definition 1 . Generically $\{x\}_{q},(q$ an integer $\geqq 0)$, will denote an expression

$$
\rho_{0}(x)+\rho_{1}(x) \log x+\cdots+\rho_{q}(x) \log ^{q} x,
$$

where the $\rho_{j}(x)$ are series, possibly divergent, of the form

$$
\rho_{j, 0}+\rho_{j, 1} x^{-1 / k}+\rho_{j, 2} x^{-2 / k}+\cdots, \quad k \text { a positive integer. }
$$

Definition 2. A curve $B$ will be said to be regular if it is simple and extends to infinity where it possesses a unique limiting direction.§

Definition 3. A region $R$ will be said to be regular if it is closed, extends to infinity, and is such that if $x$ is in $R$, then $|x| \geqq r_{1}>0$; also the boundary of $R$ is to be simple and is to consist of an arc $\gamma$ of the circle $|x|=r_{1}$ and of two regular curves extending from different extremities of $\gamma$. Generically $R\left(\theta_{1}, \theta_{2}\right)$ will designate a regular region for which the two regular curves (forming part of the boundary) have limiting directions $\theta_{1}$ and $\theta_{2}$, respectively. The number $\left|\theta_{1}-\theta_{2}\right|$ is to be termed the opening of $R$.

Definition 4 . The symbol $[x]_{q}$ will denote a function of the form $p_{0}(x)+\cdots+p_{q}(x) \log ^{q} x$ where the $p_{j}(x)$ are functions, analytic for $x \neq \infty$ in a regular region $R$, such that

† When (A) is written in a suitable form one then has the $Q_{i}(x)$ in (1.6) of the form $h_{i} x$, no logarithms will be present in (1.6a), and the series (1.6b) will be in powers of $x^{-1}$.

$¥$ With (A) taken in a suitable form, the $Q_{i}(x)$ of (1.6) will be polynomials in $x$ (of degree which may be higher than the first), no logarithms will enter in (1.6a), and the series (1.6b) will be in powers of $x^{-1}$. The coefficients of the leading powers in the $Q_{i}(x)$ are expressible in terms of the roots of the characteristic equation; the latter is of the form $E(g)=0$ where $E(g)$ is a polynomial of degree $n$, the coefficients of the various powers of $g$ being certain ones of the initial coefficients from the series (1.1).

$\S$ That is, if the equation of the curve is $\theta=f(r),(\theta=$ angle of $x ; r=|x|)$, then $\lim \theta($ as $r \rightarrow \infty)$ exists and is unique. 


$$
p_{j}(x) \sim \rho_{j}(x) \text { in } R,
$$

the $\rho_{i}(x)$ being series of the form (2.1).

One has $[x]_{q} \sim\{x\}_{q}, x$ in $R . \dagger$

It is a well known fact that when the coefficients in (A) are representable by convergent series (1.1), while in the formal solutions the polynomials $Q_{i}(x),(i=1, \cdots, n)$, are all identically zero, the formal series (1.6) will all converge (for $|x| \geqq r^{\prime} ; r^{\prime}$ sufficiently great) and will thus represent a full set of "actual" solutions of (A). The equation (A) is then said to be of Fuchsian type.

We let $B_{i, j}$ denote a regular curve along which

$$
R\left(Q_{i}(x)-Q_{j}(x)\right)=0 .
$$

Such curves will be defined only when $Q_{i}(x) \not \equiv Q_{j}(x)$. Let

$$
R_{1}, R_{2}, \cdots, R_{N}
$$

be regular regions separated by the $B_{i, j}$ curves (formed for all admissible pairs of values $i, j$ ) in such a way that interior to any such region there is no $B_{i, j}$ curve. Consider a particular region $R_{k}$ of the set (2.3). It has the form $R\left(\theta_{k, 1}, \theta_{k, 2}\right)$, where $\theta_{k, 1} \leqq \theta_{k, 2}$. The regular curves forming part of the boundary of $R_{k}$ and possessing at infinity the limiting directions $\theta_{k, 1}$ and $\theta_{k, 2}$ will be designated as $B_{k, l}$ and $B_{k, r}$, respectively.

In view of the fundamental existence theorem given in $\left(T_{1}\right)$ the following can be asserted for any fixed $k,(1 \leqq k \leqq N)$.

If $\theta_{k, 1}=\theta_{k, 2}$, the equation (A) has a full set of solutions

$$
y_{i}(x), \quad i=1, \cdots, n,
$$

whose elements $y_{i}(x)$ are analytic in $R_{k}(x \neq \infty)$, while

$$
y_{i}(x) \sim s_{i}(x), \text { in } R_{k} ; \quad i=1, \cdots, n,
$$

the series $s_{i}(x)$ being given by (1.6); that is,

$$
y_{i}(x)=e^{Q_{i}(x)} x^{r_{i}}[x]_{q_{i}} \text {, in } R_{k} ; \quad i=1, \cdots, n .
$$

If $\theta_{k, 1}<\theta_{k, 2}$, there exist regular overlapping subregions of $R_{k}$,

$$
{ }_{r} R_{k}=R\left(\theta_{k, 1}, \theta_{k, 2}\right), \quad{ }_{\imath} R_{k}=R\left(\theta_{k, 1}, \theta_{k, 2}\right),
$$

whose boundaries contain $B_{k, l}$ and $B_{k, r}^{\prime}$, respectively. $\ddagger$ So that there exist two full sets of "actual" solutions,

$\dagger$ In the sense indicated in the definitions.

$\ddagger$ The other regular curve, which forms part of the boundary of ${ }_{r} R_{k}$ (or ${ }_{l} R_{k}$ ), is interior to $R_{k}$ and has at infinity the limiting direction of $B_{k, r}^{\prime}$ (or $B_{k, l}^{\prime}$ ). The precise details are given in $\left(T_{1}\right)$. 
such that

$$
\begin{aligned}
{ }_{r} y_{i}(x) & \sim s_{i}(x), & i & =1, \cdots, n ; \text { in }{ }_{r} R_{k}, \\
{ }_{i} y_{i}(x) & \sim s_{i}(x), & i & =1, \cdots, n ; \text { in }{ }_{l} R_{k} ;
\end{aligned}
$$

that is, the functions (2.5a) are of the form $\left(\exp Q_{i}(x)\right) x^{r_{i}}[x]_{q_{i}},(i=1$, $\cdots, n)$, in the specified regions.

Results of the above description can be stated for each one of the regions (2.3). If we take a particular set of solutions relating, let us say, to $R_{1}$, the asymptotic behaviour of these solutions can be studied in the complete neighborhood of $x=\infty$ (for details, cf. $\left(T_{1}\right)$ ). $\dagger$

Under certain conditions results of the stated kind will hold in more extensive regions (see $\left(T_{1}\right)$, pp. 216-218). However, it is not to be expected that in general one could replace ${ }_{r} R_{k}$ and ${ }_{l} R_{k}$ (the regions involved in (2.5b) and (2.5c)) by their sum $R_{k}$ and assert existence of $a$ full set of "actual" solutions $y_{i}(x),(i=1, \cdots, n)$, for which $y_{i}(x) \sim s_{i}(x)$, $(i=1, \cdots, n)$, throughout $R_{k}$. In other words, no essential improvement is to be expected in this direction. The paper $\left(T_{1}\right)$ discusses also some converse problems, including the formulation of the corresponding Riemann problem.

3. Problem (B) (asymptotic methods). It is convenient to write equation (B) of $\$ 1$ in the form

$$
L(x, \lambda ; y) \equiv \sum_{k=0}^{n} \lambda^{H(n-k)} a_{n-k}(x, \lambda) y^{(k)}=0, \quad \text { integer } H \geqq 0,
$$

where the $a_{n \sim k}(x, \lambda)$ are either equal to convergent series of the form

$$
\alpha_{n-k}(x, \lambda)=\sum_{\nu=0}^{\infty} \alpha_{n-k, \nu}(x) \lambda^{-\nu}, \quad k=0,1, \cdots, n,
$$

or are asymptotic (as stated subsequent to (1.2)) to such possibly divergent series, respectively $\ddagger$ We choose the integer $H(\geqq 0)$ as small as possible. In $\S 1$ it has been assumed that in the series $\alpha_{0}(x, \lambda)$ not all the coefficients are identically zero. Hence, with $(a, b)$ properly chosen, we now may suppose that $\alpha_{0}(x, \lambda) \equiv 1$.

If $H=0$, while $a_{n-k}(x, \lambda)=\alpha_{n-k}(x, \lambda)$ (the latter series being convergent for $x$ in $(a, b)$ and for $\left.|\lambda| \geqq r^{\prime}>0\right)$, we have an analog to

$\dagger$ In general, of course, there is change of asymptotic form from curve to curve, while in the neighborhood of some of the curves the asymptotic form, figuratively speaking, may become "blurred."

$\ddagger$ The $\alpha_{n-k, \nu}(x)$ are of the same description as the $\alpha_{i, \nu}(x)$ in (1.2). 
the Fuchsian theory. As established by $\mathrm{H}$. Poincaré there exists then a full set of solutions analytic in $\lambda$ at $\lambda=\infty$ (provided the initial conditions are of the same character).

The characteristic equation for $\left(\mathrm{B}^{\prime}\right)$ is

$$
E(x ; g) \equiv \sum_{k=0}^{n} \alpha_{n-k, 0}(x) g^{k}=0 .
$$

Let $g_{i}=g_{i}(x),(i=1, \cdots, n)$, be its roots. The interval $(a, b)$ will be so chosen that the following will be true for any pair of functions $g_{i}(x), g_{j}(x),(i \neq j)$. Either $g_{i}(x)=g_{j}(x)$ for all $x$ in $(a, b)$ or $g_{i}(x) \neq g_{j}(x)$ for every $x$ in $(a, b) . \dagger$ With a proper choice of $(a, b)$, then, $\left(\mathrm{B}^{\prime}\right) \ddagger$ will possess a full set of formal solutions (1.7). The latter involve functions $Q_{i}(x, \lambda),(i=1, \cdots, n)$. Now, $Q_{i}(x, \lambda)$ will be a polynomial in $\lambda^{1 / k_{i}}$, (integer $k_{i}>0$ ). With the highest power of $\lambda$ displayed, one may write

$$
Q_{i}(x, \lambda)=q_{i, 0}(x) \lambda^{H}+\cdots
$$

here

$$
q_{i, 0}^{(1)}(x) \equiv g_{i}(x),
$$$$
i=1,2, \cdots, n \text {, }
$$

where $g_{i}(x)$ is a root of (3.2).

The number $H$ has to do with what appropriately might be called the rank of the equation (B). Whence $H$ will be termed the rank of (B).

G. D. Birkhoff gave an asymptotic theory, with the essential particulars, for the equations (B) of rank unity in the case when the roots of the characteristic equation (3.2) are distinct throughout $(a, b)$.

J. D. Tamarkin developed a theory for equations (B) of any finite rank under the assumption that the roots of the characteristic equation (3.2) are distinct in $(a, b)$.

The extension of the methods of these writers to the treatment of the more general problem, now under consideration, cannot be conveniently carried out.

In $\left(\mathrm{T}_{2}\right)$ we develop the theory of the general problem (B) (of any finite rank $H$, admitting existence of multiple roots of (3.2)). The fundamental existence theorem of $\left(T_{2}\right)$ is established with the aid of a limiting process called "iterations" akin to an analogous process which we introduced in $\left(T_{1}\right)$, suitable integrations, and factorizations.

The existence theorem of $\left(T_{2}\right)$ asserts in particular the following:

\footnotetext{
$\dagger(a, b)$ is closed.

$\ddagger$ Rather, the equation $\left(\mathrm{B}^{\prime *}\right)$ obtained by replacing the $a_{n-k}(x, \lambda)$ in $\left(\mathrm{B}^{\prime}\right)$ by the series $\alpha_{n-k}(x, \lambda)$, respectively.
} 
Let the $B_{i, j}^{1, x}$ designate curves in the complex $\lambda$-plane, extending to infinity, along which

$$
R Q_{i}^{(1)}(x, \lambda)=R Q_{j}^{(1)}(x, \lambda) \cdot \dagger
$$

There exist subintervals of $(a, b)$ such that, when $x$ is restricted to one of them, there exists a regular region $R$ in the $\lambda$-plane so that there are no curves $B_{i, j}^{1, x},(i, j=1, \cdots, n)$, interior to $R$. Let $\left(a^{\prime}, b^{\prime}\right)$ denote any particular subinterval of the kind referred to above. As a matter of notation, entailing no loss of generality, one may write

$R Q_{1}^{(1)}(x, \lambda) \leqq R Q_{2}^{(1)}(x, \lambda) \leqq \cdots \leqq R Q_{n}^{(1)}(x, \lambda), \quad x$ in $\left(a^{\prime}, b^{\prime}\right) ; \lambda$ in $R$.

Let $\alpha$ be an integer, however large. The equation (B) will possess a full set of "actual" solutions, ${ }_{\alpha} y_{i}(\lambda, x),(i=1, \cdots, n)$, with elements analytic in $\lambda,(\lambda \neq \infty)$, and continuous in $x$ for $x$ in $\left(a^{\prime}, b^{\prime}\right)$ and $\lambda$ in $R$; moreover,

$$
{ }_{\alpha} y_{i}(\lambda, x) \underset{\alpha}{\alpha} s_{i}(\lambda, x), \quad i=1, \cdots, n ; x \text { in }\left(a^{\prime}, b^{\prime}\right) ; \lambda \text { in } R,
$$

where the $s_{i}(\lambda, x)$ are the series of (1.7).

For the problem under consideration regions $R$ and full sets of "actual" solutions, of the above description, will certainly exist at least for some sufficiently small interval $\left(a^{\prime}, b^{\prime}\right)$.

Associated with the equation $\left(\mathrm{B}^{\prime}\right)$ is the system, which in matrix notation may be written as

$$
Y^{(1)}(x, \lambda)=Y(x, \lambda) D(x, \lambda), \quad Y(x, \lambda)=\left(y_{i, j}(x, \lambda)\right), \ddagger
$$

where

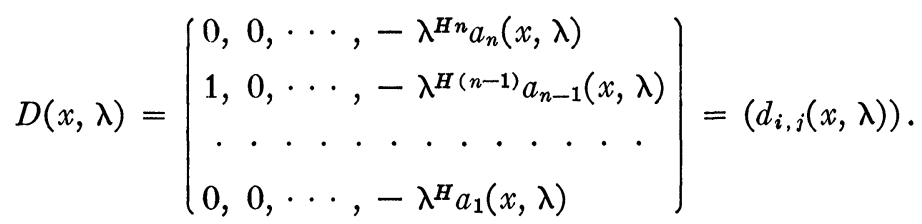

If $\left(y_{i, j}(x, \lambda)\right)$ is a matrix solution of $\left(\mathrm{B}^{\prime}\right)$, then $\left(y_{i, j}(x, \lambda)\right)$

$\dagger$ Differentiation is with respect to $x$. Whenever $Q_{i}(x, \lambda) \equiv Q_{j}(x, \lambda)$ the convention will be that there are no corresponding $B_{i, j}^{1, x}$ curves. Sufficiently far from the origin the $B_{i, j}^{1, x}$ curves are simple; moreover, at infinity they will possess limiting directions. A particular curve (3.4) will depend on $x$. As $x$ varies in the interval this curve may vary; the angle of the sector within which this variation takes place can be made as small as desired by a suitable choice of the interval for $x$.

$\ddagger\left(y_{i, j}(x, \lambda)\right)$ is a matrix of $n^{2}$ elements, with $y_{i, j}(x, \lambda)$ in the $i$ th row and $j$ th column $(i, j=1, \cdots, n)$. 
$=\left(y_{i, 1}^{(j-1)}(x, \lambda)\right)$, and the $y_{i, 1}(x, \lambda),(i=1, \cdots, n)$, will constitute a full set of solutions of $\left(\mathrm{B}^{\prime}\right)$. If the $y_{i}(x, \lambda)\left(=y_{i, 1}(x, \lambda) ; i=1, \cdots, n\right)$ form a full set of solutions of $\left(\mathrm{B}^{\prime}\right)$, the matrix $Y(x, \lambda)=\left(y_{i}^{(j-1)}(x, \lambda)\right)$ will satisfy $\left(B^{\prime \prime}\right)$. More generally, there exists a type of reciprocal relationship between a matrix system

$$
Y^{(1)}(x, \lambda)=Y(x, \lambda) A(x, \lambda),
$$$$
A(x, \lambda)=\left(a_{i, j}(x, \lambda)\right),
$$

where the $a_{i, j}(x, \lambda),(i, j=1, \cdots, n)$, are functions of the same type as the $a_{i}(x, \lambda)$ in (B), ( $\left.\$ 1\right), \dagger$ and a single equation (B). The system $\left(\mathrm{B}^{\prime \prime \prime}\right)$ is satisfied by a formal matrix $S(x, \lambda)=\left(s_{i, j}(x, \lambda)\right)=\left(\sigma_{i, j}(x, \lambda)\right.$ $\left.\cdot \exp Q_{i}(x, \lambda)\right)$, where the series $\sigma_{i, j}(x, \lambda),(i, j=1, \cdots, n)$, are of the same description as the $\sigma_{i}(x, \lambda)$ of $(1.7 \mathrm{a})$. The elements of a row will constitute a formal solution.

Under conditions and with the notation similar to that under which (3.5) has been asserted the following can be stated:

There exists an "actual" matrix solution of $\left(\mathrm{B}^{\prime \prime \prime}\right),{ }_{\alpha} Y(x, \lambda)$, such that

$$
{ }_{\alpha} Y(x, y) \underset{\alpha}{\sim} S(x, \lambda), \quad x \text { in }\left(a^{\prime}, b^{\prime}\right) ; \lambda \text { in } R .
$$

Such a matrix can be constructed for $\alpha=1,2, \cdots$.

Any matrix $Z(x, \lambda)={ }_{\alpha} Y^{-1}\left(a^{\prime}, \lambda\right){ }_{\alpha} Y(x, \lambda)$ (or $\left.{ }_{\alpha} Y^{-1}\left(b^{\prime}, \lambda\right)_{\alpha} Y(x, \lambda)\right)$ will be independent of $\alpha$ and will, of course, satisfy the system ( $\left.\mathbf{B}^{\prime \prime \prime}\right)$. As pointed out in $\left(\mathrm{T}_{2}\right), Z(x, \lambda)$ will satisfy certain asymptotic relations in the ordinary sense $\ddagger$ for $x$ on $\left(a^{\prime}, b^{\prime}\right)$ and $\lambda$ in $R$.

In $\left(\mathrm{T}_{2}\right)$, pp. $40-44$, are also made applications to integro-differential equations of the form

$$
L(x, \lambda ; y(x, \lambda))=a(x, \lambda)+\int_{a^{\prime}}^{x} b(u, x, \lambda) y(u, \lambda) d u,
$$

where $L$ is the differential operator of the left member of (B), ( $\$ 1)$.

On the basis of our existence results some applications are also made to boundary value problems. $\S$ For the general problem (B), however, development of an adequate boundary value theory (leading to expansions of arbitrary functions) apparently necessitates

$\dagger$ It is assumed that the determinant $|A(x, \lambda)| \not 0$. This relationship is not obvious but is to be expected and is established without much difficulty.

$\ddagger$ Cf. $\left(\mathrm{T}_{2}\right)$, p. 33 .

$\S \mathrm{A}$ formulation of general boundary value problems, together with a number of significant results, is given by J. D. Tamarkin, Mathematische Zeitschrift, loc. cit. A treatment of boundary value problems for linear systems, with the parameter entering linearly, is due to Birkhoff and Langer, Proceedings of the American Academy of Arts and Sciences, loc. cit. 
some restrictions with respect to the character of the polynomials $Q_{i}(x, \lambda),(i=1, \cdots, n)$. Thus, it appears, we would have to assume that the various regions for which (B) has solutions of known asymptotic form (as implied by the existence theorem of $\left(\mathrm{T}_{2}\right)$ ) abut on each other.

4. Problem (A) (Laplace integrals). The equation (A), ( $\$ 1)$, will be now assumed to possess coefficients representable by convergent series of the form (1.1). By means of a transformation $x^{\alpha}=x_{1}(\alpha$ suitably chosen) the equation (A) is brought to the form

$$
L(y) \equiv \sum_{k=0}^{n} d_{n-k}(x) y^{(k)}=0,
$$

where we wrote $x$ without the subscript; here $\dagger$

$$
d_{n-k}(x)=\sum_{\nu=0}^{\infty} d_{n-k, \nu} x^{-\nu / p}, \quad \text { integer } p>0 ; d_{0,0} \neq 0,
$$

all the series being convergent for $|x| \geqq r^{\prime}(>0)$. The characteristic equation of $\left(A^{\prime}\right)$ will be

$$
E(\rho) \equiv \sum_{k=0}^{n} d_{n-k, 0} \rho^{k}=0 .
$$

Horn's results, $\ddagger$ as stated for $\left(\mathrm{A}^{\prime}\right)$, are as follows:

Under the hypothesis that the roots of the characteristic equation (4.2) are distinct, $\left(\mathrm{A}^{\prime}\right)$ possesses a full set of solutions of the form

$$
e^{Q_{i}(x)} x^{r i} \eta_{i}(x), \quad i=1,2, \cdots, n,
$$

where the $Q_{i}(x)$ are polynomials in $x^{1 / p}$ and the $\eta_{i}(x)$ are of the form

$$
\eta_{i}(x)=\eta_{i, 0}+\sum_{\nu=1}^{p} x^{1-\nu / p} \eta_{i, \nu}(x)
$$

the $\eta_{i, \nu}(x),(\nu=1, \cdots, p ; i=1, \cdots, n)$, being convergent factorial series of the type

$$
\eta_{i, \nu}(x)=\sum_{s=0}^{\infty} \frac{a_{s}^{i, \nu}}{x(x-\gamma) \cdots(x-s \gamma)} .
$$

Here $|\gamma|$ must be sufficiently great and $\gamma$ is the same for all solutions. Moreover, $\angle \gamma$ is allowed to have any value except certain ones,

$\dagger p$ generally will be different from the integer so denoted in (1.1).

$¥$ We state them in a form slightly different from that of Horn. 
depending on the roots of (4.2). $\dagger$ Formally, solutions (4.2) are compatible with the corresponding formal solutions.

Our treatment of $\left(\mathrm{A}^{\prime}\right)$ along these lines, as given in $\left(\mathrm{T}_{3}\right) \ddagger$ goes further. The pertinent results from $\left(\mathrm{T}_{3}\right)$ will be forthwith stated.

In accordance with facts pointed out in $\$ 1$, equation $\left(\mathrm{A}^{\prime}\right)$ has a full set of formal solutions $s_{i}(x)=x^{r i} \sigma_{i}(x) \exp Q_{i}(x),(i=1, \cdots, n)$, where the $\sigma_{i}(x)$ are of the form (1.6a), and the $Q_{i}(x)$ are polynomials in $x^{1 / k_{i}}$, while the coefficients of the powers of $\log x$ in the $\sigma_{i}(x)$ are power series in $x^{-1 / k_{i}}\left(k_{i}\right.$ integers). It will be said that $s_{i}(x)$ is normalई when $k_{i}=p$. When $k_{i}=r_{i}{ }^{\prime} p$ (integer $r_{i}{ }^{\prime}>1$ ) the corresponding formal solution $s_{i}(x)$ will be said to be anormal. Consider a root $\rho$ of $(4.2)$ of multiplicity $\phi(\geqq 1)$. If $\phi=1$, the corresponding formal solution will be normal and will contain no powers of $\log x$. If If $\phi>1$, the following cases may present themselves:

CASE 1 . The $\phi$ polynomials $Q_{i}(x)$ from the formal solutions corresponding to the root $\rho$ of (4.2) are not all identical.

CASE 2. This is the alternative to the previous case.

In Case 2 the formal solutions corresponding to the root $\rho$ are all normal.

The theorem (for differential equations) proved in $\left(\mathrm{T}_{3}\right)$ can be stated as follows:

Consider a root $\rho_{1}$, of multiplicity $\phi$, of the characteristic equation $E(\rho)=0$ (see (4.2)) associated with $\left(\mathrm{A}^{\prime}\right)$. Assume that with respect to this root Case 2 takes place. As a matter of convenience, entailing no loss of generality, we may take $\rho_{1}=0$. For every $\bar{t}$, not coincident with a value of an angle of a non-zero root of $E(\rho)=0$, the following will be true.

$\left(\mathrm{A}^{\prime}\right)$ possesses $a$ set of $\phi$ (linearly independent) solutions

$$
\begin{array}{r}
y_{j}(x)=e^{Q(x)} x^{r} \sum_{h=0}^{j-1} \log ^{h} x\left[{ }_{h} \eta_{0^{j-1}}+\sum_{\nu=1}^{p} x^{(p-\nu) / p}\right. \\
\left.\cdot \sum_{s=0}^{\infty} \frac{{ }_{h} A_{\nu, s}^{j-1}(\bar{t})}{x(x-\gamma) \cdots(x-s \gamma)}\right], \\
j=1, \cdots, \phi ; Q(x)=\text { polynomial in } x^{1 / p},
\end{array}
$$

where $|\gamma|$ is suitably great and $\angle \gamma=\bar{\gamma}=-\bar{t}$; the factorial series in (4.3) will all converge in a half-plane

$$
R\left(e^{i \bar{t}} x\right)<-q(<0) .
$$

$\dagger$ For a given admissible $\gamma$ the series $(4.2 \mathrm{~b})$ will converge in a certain half-plane. $\ddagger$ W. J. Trjitzinsky, Transactions of this Society, loc. cit.

$\S$ This is the value $p$ involved in (4.1).

I This is the case treated by Horn. 
The implications of this theorem for (A), (\$1) are obvious. The $\phi$ corresponding solutions of (A) will be of the form (4.3), with $x$ replaced by a certain power of $x$; as a consequence the series involved in these solutions will converge in certain sectors.

The above process constitutes, of course, a process of summing the corresponding formal series solutions.

The theorem is of greatest possible completeness in the sense that even normal formal series solutions do not always lead to convergent factorial series developments, if, corresponding to the multiple root in question, the polynomials $Q_{i}(x)$ are not all identical. This point is made clear with the aid of the following example.

The equation

$$
L_{3}(y) \equiv y^{(3)}+a x^{-1} y^{(1)}+d x^{-3} y=0, \quad a \neq 0, d \neq 0,
$$

is of the form $\left(\mathrm{A}^{\prime}\right)$; its characteristic equation has a root, $\rho=0$, of multiplicity three. There is a formal solution

$$
y(x)=\sum_{\nu=0}^{\infty} y_{\nu} x^{-\nu}, \quad y_{0}=1,
$$

where

$$
y_{\lambda+1}=q(0) q(1) \cdots q(\lambda), \quad q(\lambda)=\frac{d}{a(\lambda+1)}-\frac{\lambda(\lambda+2)}{a} .
$$

The power series (4.4a) diverges so fast that exponential summability fails, and nothing of the type stated in our theorem will hold in connection with this formal solution. $\dagger$

We shall outline very briefly the steps used in deriving the theorem referred to above. We apply to $\left(\mathrm{A}^{\prime}\right)$ the transformation

$$
y_{j}(x)=e^{Q(x)} x^{r} \sum_{h=0}^{j-1} \log ^{h} x\left({ }_{h} \eta_{0}^{j-1}+\sum_{\nu=1}^{p} x^{(p-\nu) / p_{h} \eta_{\nu}^{j-1}(x)}\right) .
$$

This yields a differential system

$$
\begin{aligned}
& \sum_{\beta=0}^{j-h-1} \sum_{k=0}^{n} \sum_{\zeta=1}^{p}{ }_{\beta} a_{\zeta, k}^{h, \nu}(x)_{h+\beta} \eta_{\zeta}^{j-1(k)}(x)=g^{h, \nu, j}(x), \\
& h=0, \cdots, j-1 ; \nu=1, \cdots, p,
\end{aligned}
$$

where the character of the coefficients is determined in detail $\left(\left(\mathrm{T}_{3}\right)\right.$, p. 96). On writing $t=|t| e^{i \bar{t}}$, we suppose integrals

$\dagger$ In fact, it appears, as well, that no other method of summation will be applicable in this case. 


$$
\int_{0}^{\infty}, \quad \int_{0}^{t}
$$

to be extended over the ray $(0, \infty)$, of angle $\bar{t}$, denoted by $I(\bar{t})$, and the rectilinear segment $(0, t)$, respectively. If the variable $x$ is so restricted that $\lim _{t}\left|e^{t x} t^{\alpha}\right|=0$ (every $\alpha>0 ; t \rightarrow \infty$ along $I(\bar{t})$ ), then, as is well known, we have formally

$$
a(x)=\sum_{s=1}^{\infty} a_{s} x^{-s}=\int_{0}^{\infty} \tilde{a}(t) e^{t x} d t
$$

where

$$
\tilde{a}(t)=\sum_{\nu=0}^{\infty} \tilde{a}_{\nu} t^{\nu-1}, \quad \tilde{a}_{\nu}=\frac{(-1)^{\nu} a_{\nu}}{(\nu-1) !} .
$$

On the basis of these facts Laplace integrals are introduced by means of the following transformations (refer to (4.5))

$$
\begin{aligned}
& { }_{h} \eta_{\nu}^{j-1}(x)=\int_{0}^{\infty} h \tilde{\eta}_{\nu}^{j-1}(t) e^{t x} d t, \\
& h=0, \cdots, j-1 ; j=1, \cdots, \phi ; \nu=1, \cdots, p .
\end{aligned}
$$

This transformation, applied to (4.6), yields a system of integral equations

$$
\begin{array}{r}
t_{h} \tilde{\eta}_{\nu}^{j-1}(t)=\sum_{\beta=0}^{j-h-1} \sum_{\zeta=1}^{p} \int_{0}^{t} \beta^{*} c_{\zeta}^{h, \nu}(t, \tau)_{h+\beta} \tilde{\eta}_{\zeta}^{j-1}(\tau) d \tau+{ }^{*} g^{h, \nu, j}(t), \\
h=0, \cdots, j-1 ; \nu=1, \cdots, p .
\end{array}
$$

The character of the coefficients of this system is specified in $\left(T_{3}\right)$, Lemma 3 (p. 103). In proving convergence of the formal solutions $\dagger$ ${ }_{h} \tilde{\eta}_{S}^{j-1}(t)$, satisfying (4.8), the method of successive approximations (used with success by Horn in his more restricted problem) leads to apparently unsurmountable algebraic difficulties. The method pursued in $\left(\mathrm{T}_{3}\right)$ to overcome this difficulty was to establish a "dominant" system of integral equations; that is, a system from the convergence of whose formal solutions convergence of a set of solutions of (4.8) can be inferred. Such a system, as well as proof of convergence of the formal series solutions, is given in $\left(T_{3}\right)$, pp. 103-113.

In order that the integrals (4.7) should lead to convergent factorial

$\dagger$ Power series in $t$. 
series expansions, $\dagger$ in view of certain fundamental developments due to $\mathrm{N}$. E. Nörlund, $¥$ it is necessary to prove that

$$
\left|{ }_{h} \tilde{\eta}_{\nu}{ }^{j-1}(t)\right|<C e^{q|t|}, \quad h=0,1, \cdots, j-1 ; \nu=1, \cdots, p,
$$

along every ray $\bar{t}(=\angle t)$ in $P_{0}(C$ and $q$ independent of $\bar{t}$ and $|t|$, positive and sufficiently great). Here $P_{0}$ is the complex $t$-plane, excluding a neighborhood of $t=0$ and excluding small sectors, each with vertex at $t=0$, and containing the zeros of $d_{0,0} t^{n-\phi}+d_{1,0} t^{n-\phi-1} \ldots$ $+d_{n-\phi, 0}=0 \S$ in their interiors. The inequalities (4.9) have been established in $\left(\mathrm{T}_{3}\right)$, pp. 114-116, following lines somewhat similar to those employed by Horn in his proof of analogous inequalities. The existence theorem stated in connection with (4.3) is inferred with the aid of (4.9).

5. Some general remarks. In connection with the equation (B), (\$1) a remaining problem of considerable importance is that regarding the behaviour of solutions in the neighborhood of isolated points $x_{0}$ (on some real interval to which $x$ is restricted) at which a number of roots $g_{i}(x)$ of the characteristic equation (3.2) coincide. We may have two intervals,

$$
\left(u, x_{0}\right), \quad\left(x_{0}, l\right), \quad u<x_{0}<l,
$$

such that the two full sets of formal solutions, corresponding to these intervals, are essentially distinct from each other. Development of a comprehensive theory covering this situation is highly desirable.

Methods of the type of those introduced in $\left(T_{1}\right)$ and $\left(T_{2}\right)$ (in particular, the processes of "iterations") appear to be destined to be of utility in many similar problems. $\|$

In conclusion it would be appropriate to mention that the results established in $\left(T_{1}\right)$ and $\left(T_{2}\right)$ have found effective application in the field of non-linear differential equations. $\uparrow$

UNIVERSITY OF ILLINOIS

$\dagger$ And in fact to secure convergence of these integrals.

$\ddagger$ N. E. Nörlund, Leçons sur les Séries d'Interpolation, Paris, 1926, pp. 206-208. Also see his theorem on p. 203.

$\S$ Here $d_{n-\phi, 0} \neq 0$. Moreover, by a transformation, $x^{r} \exp Q(x)$ is supposed to be reduced to unity.

\| In this connection should be mentioned some significant papers by C. Hurd (not yet published) in which such methods were applied to equations with several parameters.

I W. J. Trjitzinsky, Theory of non-linear singular differential systems, Transactions of this Society, vol. 42 (1937), pp. 225-321. 